50 DISCUSSIONS ON ADVANCE CARE PLANNING - AS SPECIALISTS, ARE WE CONFIDENT IN TACKLING THIS SUBJECT?

Dawn Matthews, Emer McKenna, Julie Gater, Melanie Sheard. Douglas Macmillan Hospice, Stoke-on-Trent

\subsection{6/bmjspcare-2018-ASPabstracts.77}

Background The Advance Care Planning Procedure approved by the Clinical Governance Committee states 'All patients will be given the opportunity to plan their future care.' The aims of the procedure are to:

- Provide a consistent approach to advance care planning across all service areas

- Ensure that the Advance care plan (ACP) is considered when the patient moves between services

- Ensure the ACP is reviewed as the patient's condition changes.

- Ensure that staff understand what can and cannot be included in an ACP

In accordance with the requirements of the CQUIN, this audit aims to monitor the proportion of patients who were offered the opportunity to discuss advanced care planning.

Methods 30 community based patients, 5 patients from the day therapy unit and 10 patients from the inpatient unit were randomly selected by the administration team. Inclusion criteria were for patients to have been seen at least 3 times, and for the patient to have capacity.

A retrospective review of the medical notes was conducted and details of ACPs were recorded, using a pre-determined audit questionnaire.

Results Initial results have shown that $80 \%$ of ACP discussions were commenced by the first hospice service assessing the patient, and the remaining $20 \%$ had these discussions on contact with a second service. $46.7 \%$ were discussed during routine assessments. Discussions about preferred place of death $(72 \%)$ and resuscitation (76\%) occurred more frequently than those about Lasting Power of Attorney (33.3\%). Of the patients who had died, $61.5 \%$ of ACPs were reviewed in the month before their death.

Conclusions This audit showed that Advance Care Planning is being discussed with patients and considered when moving between services. Further training is needed to ensure ACPs are reviewed as a patient's condition changes and that all aspects of the ACP are discussed and documented.

\section{TO COMFORT ALWAYS: INTRODUCING A NOVEL METHOD OF RECORDING APPROPRIATE PHYSIOLOGICAL OBSERVATIONS FOR PATIENTS AT THE END OF LIFE IN A UNIVERSITY TEACHING HOSPITAL IN ENGLAND}

Oliver Emmerson, Jon Tomas. University Hospitals Birmingham NHS Foundation Trust, University of Birmingham

\subsection{6/bmjspcare-2018-ASPabstracts.78}

Introduction Collection of vital signs is a deeply ingrained part of hospital care and culture. Routine measurements in dying patients, however, are often superfluous and can be uncomfortable. QEHB patients in their last hours-to-days of life can be assigned to 'Comfort Observations' (CO) electronically. When initiated, an icon is displayed on the Trust's observations recording system, suggesting only nominal observations be taken.

Objective To assess the feasibility of using electronic $\mathrm{CO}$ as a component of End of Life Care (EoLC) for acute hospital inpatients.

Methods The records of QEHB medical/oncology/haematology patients who died between 1/6/2017 and 31/08/2017 were accessed and the following data recorded: demographics; dates of: admission, CO initiation, death; evaluation of observations taken; prescribed medications. Numerical data was tabulated and analysed in Microsoft Excel.

Results 260 patients from the specified specialties died in the three-month period. 127 (49\%) had CO initiated at the time of death, of which $74(58 \%)$ experienced an inappropriate level of observation measurement. Mean length of admission: 11.20 days (1-69). Mean time between CO initiation and death: $2.86(0-33)$ days. 30 patients (24\%) were receiving intravenous antibiotics at the time of death.

Discussion Not all deaths were anticipated or of a nature such that CO should have been instituted. Nonetheless, when used, the mean time between starting $\mathrm{CO}$ and death suggests appropriate initiation. In contrast, only a minority of patients on $\mathrm{CO}$ had them done in accordance with Trust guidance. It is further concerning that a sizeable proportion of patients expected to die were receiving intravenous antibiotics.

Conclusion Our electronic CO system is a novel and viable method of initiating and recording observations for inpatients at the end of life. Despite this demonstrated reduction in personal burden it still remains to be seen what the effect of $\mathrm{CO}$ is on overall quality of EoLC.

\section{DECISIONS ABOUT CARDIOPULMONARY RESUSCITATION IN PATIENTS RECEIVING SPECIALIST PALLIATIVE CARE: A MULTI-CENTRE REGIONAL AUDIT OF PRACTICE}

Joanna Roberts, Rachel McDonald, Claire Cadwallader, Kate Marley, Jenny Smith, Seamus Coyle, Kate Gratwick, Anthony Thompson. Woodlands Hospice, Royal Liverpool University Hospital, Whiston Hospital, Clatterbridge Cancer Centre, Countess of Chester, Willowbrook Hospice

\subsection{6/bmjspcare-2018-ASPabstracts.79}

Background When performed inappropriately cardiopulmonary resuscitation (CPR) can prevent those with irreversible illness having a peaceful death. In people approaching the end of life, making decisions about whether to attempt CPR is integral to good care.

Aim

- Audit clinical practice around CPR decision-making and communication with patient's, those important to them and other healthcare professionals (HCPs) against regional standards.

- Use audit outcomes to update regional standards and guidelines.

Methods

- Systematic literature review examining education for HCPs, communication with patients and those important to them and dissemination of decisions

- Survey of HCPs working in specialist palliative care

- Multi-centre retrospective case note review of patients with a do not attempt cardiopulmonary resuscitation (DNACPR) 